\title{
Comorbilidad entre síndrome de Burnout, depresión y ansiedad en una muestra de profesores de Educación Básica del Estado de México
}

\author{
Francisco Sánchez-Narváez y Juan Jesús Velasco-Orozco \\ Universidad Estatal del Valle de Ecatepec, México/Instituto \\ de Ciencias de la Educación del Estado de México, México
}

\section{Resumen}

El objetivo de esta investigación fue evaluar la prevalencia y comorbilidad entre síndrome de burnout, la depresión y la ansiedad, si se manifiestan por separado el uno del otro o están estrechamente entrelazados de forma que refleje el mismo fenómeno. Se realizó un estudio transversal que incluye 459 (61 por ciento mujeres) maestros. El burnout se evaluó con el Maslach Burnout Inventory para educadores. La depresión y la ansiedad se evaluó con los Inventarios de Beck de Depresión y Ansiedad. Las tasas de prevalencia del burnout (20.8 por ciento), depresión (10.6 por ciento) y ansiedad (30.9 por ciento) son altas. La comorbilidad entre ellos es de 19.7 por ciento. Los maestros con burnout presentan incremento en depresión y ansiedad. Además, el agotamiento emocional fue significativamente asociado con la ansiedad y la depresión más que con la despersonalización o realización personal.

Palabras clave: Profesores, síndrome de Burnout, ansiedad y depresión.

Abstract

Comorbidity between burnout syndrome, depression and anxiety in a Elementary School teachers sample, State of Mexico

The aim of this research was to examine the prevalence and comorbidity between burnout syndrome, depressive and anxiety, whether manifest themselves separately from each other or are so closely intertwined as to reflect the same phenomenon. A cross-sectional study involving 459 (61 por ciento female) Mexican schoolteachers was conducted. Burnout syndrome was assessed with the Maslach Burnout Inventory Educators. Depression and anxiety was assessed with the Inventories Beck Depression and Anxiety. Prevalence rates of burnout (20.8 por ciento), depression ( 10.6 por ciento) and anxiety (30.9 por ciento) are high. Comorbidity among them is 19.7 por ciento. Teachers with increasing burnout experienced increases in depression or anxiety. In addition, emotional exhaustion was significantly associated with anxiety and depression than with depersonalization or personal accomplishment. Our results are consistent with findings showing qualitative and quantitative symptom overlap of exhaustion, depression and anxiety.

Key words: Teachers, Burnout syndrome, anxiety and depression. 


\section{INTRODUCCIÓN}

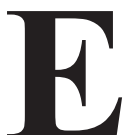

n América Latina como en otros países, los cambios económicos, las transformaciones sociales y la extensión de la longevidad de la población han modificado el panorama de la salud de la población en general, con ello, el incremento de los problemas de índole psicosocial como el síndrome de burnout y las enfermedades mentales. Los estudios epidemiológicos realizados en la última década indican que existe un aumento de estos trastornos. La salud mental de los maestros juega un papel muy importante en la calidad educativa y en los procesos que se desarrollan relacionados a la enseñanza-aprendizaje afectando no solo a los maestros sino también a los alumnos (Claro y Bedegral, 2003). También influyen en otros aspectos donde la asistencia y participación de los maestro es importante afectando la estructura académica, organizacional y administrativa, ya que este tipo de enfermedades genera un alto índice de ausentismo, incapacidad laboral y jubilación prematura.

Uno de los trastornos que afecta a los profesores en forma significativa es el síndrome de burnout el cual se presenta como una respuesta al estrés crónico. Éste fue descrito por primera vez por Herbert Freudenberger en 1974 quien lo definió como un estado de fatiga o frustración que se produce por la dedicación a una causa o forma de vida, que no produce la suficiente gratificación o esfuerzo (Freudenberguer, 1974).

Años más tarde, el burnout fue descrito como un síndrome de agotamiento e indiferencia que ocurre en trabajadores que ofrecen servicios o brindan ayuda a personas, el cual presenta tres dimensiones: i) agotamiento emocional: en esta situación, la persona siente que ya no puede dar más de sí misma en el ámbito afectivo o psicológico; ii) la despersonalización: referido al desarrollo de actitudes negativas y de insensibilidad hacia los colegas, los clientes o receptores de los servicios, se asocia con una actitud un tanto cínica e impersonal y de aislamiento hacia los demás y; iii) disminución de realización personal: que lleva a una percepción de que las posibilidades de logro en el trabajo han desaparecido, junto con vivencias de fracaso y sentimientos de baja autoestima (Maslach y Jackson, 1981 y 1996).

Los reportes en la literatura a nivel mundial lo consideran como uno de los principales síndromes que afecta la salud de los trabajadores, siendo la docencia la segunda con mayor prevalencia 27 por ciento solo después de las profesiones dedicadas a los servicios de salud (Schaufeli y Enzmann, 
1998). En México estos datos en la población trabajadora son controversiales debido a que se han utilizado distintos instrumentos para evaluarlo y los estudios en la población de los maestros son escasos (Juárez-García et al., 2014).

Existe una amplia literatura que da cuenta del síndrome del Burnout y los factores laborales que influyen en el desarrollo de éste (Aronsson et al., 2017). Sin embargo, en México es insuficiente cuando se aborda la interacción de los tres trastornos (Burnout, depresión y ansiedad) presentes en la población de docentes. La literatura al respecto en otros países sugiere que uno de cada 10 trabajadores de los estados industrializados sufre depresión, ansiedad, estrés o síndrome de Burnout. En el Reino Unido el departamento de salud y la confederación de la industria británica estiman que alrededor de 15 a 30 por ciento puede presentar un algún problema en salud mental durante su vida laboral. En este país es la principal causa de enfermedad e incapacidad laboral (OIT, 2000). En términos generales se estima que alrededor de 20 por ciento de la población adulta trabajadora en los países de la Comunidad Europea presenta algún problema de salud mental en algún momento de su vida. En Estados Unidos de Norteamérica se presenta un panorama similar siendo los trastornos depresivos los más comunes de salud en la población trabajadora (OIT, 2000).

En México existen pocos estudios que evalúen las enfermedades mentales más frecuentes en la población y en particular por riesgo laboral, como por ejemplo, la docencia. Se estima en nuestro país una prevalencia en la población de depresión de 6.2 a 8.1 por ciento y de los trastorno de ansiedad generalizada 5.5 por ciento (Kohn et al., 2005). Sin embargo, en la literatura a nivel mundial se han encontrado prevalencias más alta en la población de maestros, en Inglaterra 23 por ciento presenta depresión y 37 por ciento ansiedad (Chambers y Belcher, 1993). Así como 19.4 por ciento presenta síntomas de depresión entre moderado y severo (Kidger et al., 2016). En Italia se reportan también prevalencias mayores a 49 por ciento con depresión y 11 por ciento con ansiedad (Borrelli, Beneve, Fiorilli, D'Amelio y Pozz1, 2014). En Hong Kong se estima una prevalencia de ansiedad de 75.8 por ciento (Chong, 2010) o en Francia en donde se reportan prevalencias en cualquier desorden o tipos de ansiedad (fobias, desórdenes de pánico o trastornos obsesivos compulsivos) en maestras 16.4 por ciento mayor que en maestros 7.7 por ciento y trastornos del estado de ánimo de 44.6 y 25.6 por ciento, respectivamente (Kovess-Masfésty et al., 2006).

Los estudios en México sobre síndrome de Burnout (SBO) en profesores se centran principalmente en estudiar algunos factores psicosociales 
entre ellos el estrés como su causa (Galvan et al., 2010; Barraza-Macias, 2011; Pando Moreno et al., 2006). Con respecto a otros grupos de riesgo como médicos y enfermeras existen algunos estudios que abordan el análisis del SBO, la depresión y la ansiedad (Oliveira, Pereira, 2012; Lindwall, Gerber, Jonsdottir y Börjenson, 2014; Bianchi, Verkuilen, Brisson, Schonfeld y Laurent, 2016). En estos se identifica de forma independiente la presencia de los trastornos pero no evalúan su interacción. Otra de las dificultades presentes en estas publicaciones es que abordan el estudio del Burnout, así como la depresión y la ansiedad con diferentes instrumentos y conceptos por lo que la comparabilidad entre estos datos presentan un sesgo importante (Juárez-García et al., 2014).

De acuerdo con Caballero-Domínguez, González-Gutiérrez y PalaciosSañados, 2015; Pereira-Lima y Loureiro, 2015; Lopes Cardozo et al., 2012, existe una relación entre el Burnout con la presencia de depresión y ansiedad. También, se ha documentado que las personas con Burnout tienen doce veces mayor riesgo de presentar depresión y hasta trece veces más de ansiedad (Perales, Chue, Padilla y Barahona, 2011). Lo anterior nos sugiere la asociación entre estos trastornos de forma significativa.

Sin embargo, la relación y diferencia del SBO con éstas enfermedades se ha visto limitada por la definición y los criterios para clasificarlo, por lo que la investigación empírica resulta insuficiente y contradictoria en algunos casos (Fischer y Boer, 2011).

Las enfermedades mentales y psicosomáticas son más frecuentes en los maestros que en otros profesionales y presentan un mayor número de malestares como agotamiento, fatiga, dolor de cabeza y tensión cuyo origen de estos padecimientos es muy inespecífico (Scheuch, Eva y Seibt, 2015).

Los límites entre el síndrome de Burnout, depresión y ansiedad no son muy claros, incluso proponer una delimitación rigurosa podría resultar muy arbitraria (Maslach Schaufeli, 1993) ya que desde el punto de visto clínico comparten síntomas muy semejantes; entre las características que comparten podemos encontrar fatiga, irritabilidad, deterioro en el desempeño, sensación de angustia en el trabajo y vida personal. También, se presenta ausentismo laboral, disminución de su actividad física, deterioro de la atención y la memoria, entre otros (Feuerhahn et al., 2008; Talarowska et al., 2015; Marin et al., 2011).

Estas diferencias son tan sutiles que de acuerdo a Schaufeli (1999), las diferencia entre estrés y Burnout por ejemplo, es solo en el proceso, el estrés es algo continuo que experimenta el profesionista y el Burnout es el resultado final o consecuencia del estrés. 
Así también, la relación entre depresión y Burnout son dos estados independientes en el que la depresión es considerada como un estado emocional generalizado y en el caso del Burnout es dependiente del trabajo aunque también pueden presentarse ambos o sobreponerse (Schaufeli, 1999; Maslach y Schaufeli, 1993; Bianchi, Schonfeld, Laurent 2015b). Incluso algunos autores señalan que el Burnout puede ser considerado como un subtipo de depresión más que otro trastorno (Bianchi, Schonfeld, Laurent, 2015a).

Es importante señalar que el síndrome de Burnout y los otros trastornos mentales como la depresión o la ansiedad son entidades distintas pero que pueden afectar o coexistir simultáneamente en los trabajadores. En México faltan estudios que aborden estas enfermedades de forma individual y las comorbilidades que pueden presentarse en cada una de ellas. Por lo que la finalidad de la presente investigación es describir y estimar la comorbili$\mathrm{dad}^{21}$ del síndrome de Burnout, depresión y ansiedad en una muestra de profesores de educación básica del Estado de México.

\section{MÉTodo}

\section{Población}

La población incluida para participar en este estudio se seleccionó de una muestra no aleatorizada de profesores que laboran en escuelas públicas adscritos a la Subsecretaría de Educación Básica y Normal del Estado de México, pertenecientes a la Subdirección Regional de Amecameca y que trabajan en el municipio de Valle de Chalco Solidaridad en los niveles de primaria y secundaria.

La distribución por número de profesores en este municipio y niveles educativos fue de 1211 de los cuales, 616 tienen su plaza principal en primarias y 515 en secundaria. El tamaño de la muestra se calculó a partir de la siguiente fórmula.

$$
\begin{aligned}
& \mathrm{n}=\frac{\mathrm{N} \times \mathrm{Z}^{2}(\mathrm{p} \times \mathrm{q})}{\mathrm{d}^{2} \times(\mathrm{N}-1)+\mathrm{Z}^{2} \times(\mathrm{p} \times \mathrm{q})} \\
& \text { Donde, } \quad \mathrm{n}=\frac{1211 \times(1.96)^{2}(0.5 \times 0.5)}{(0.035)^{2} \times(1211-1)+(1.96)^{2} \times(0.5 \times 0.5)}= \\
& \text { Despejando la formula } \\
& \mathrm{n}=\quad \frac{1211 \times 3.84 \times 0.25}{0.00123 \times 1210+3.84 \times 0.25}=\frac{1162}{2.44}=476
\end{aligned}
$$

\footnotetext{
${ }^{21}$ La comorbilidad es entendida como la existencia de un trastorno principal en presencia de uno o más padecimientos secundarios.
} 
El número de profesores que se calculó para el muestreo fue 476 es decir, 39.3 por ciento de la población total (1211) con una precisión de 3.5 por ciento. A partir de los criterios de inclusión, exclusión y eliminación se incrementó el tamaño de la muestra a ocho por ciento (38 profesores), es decir, se consideró un total de 514 maestros correspondientes a los niveles de primaria y secundaria que representa 42.2 por ciento del total de los docentes (Figura 1).

Figura 1: Diagrama de flujo

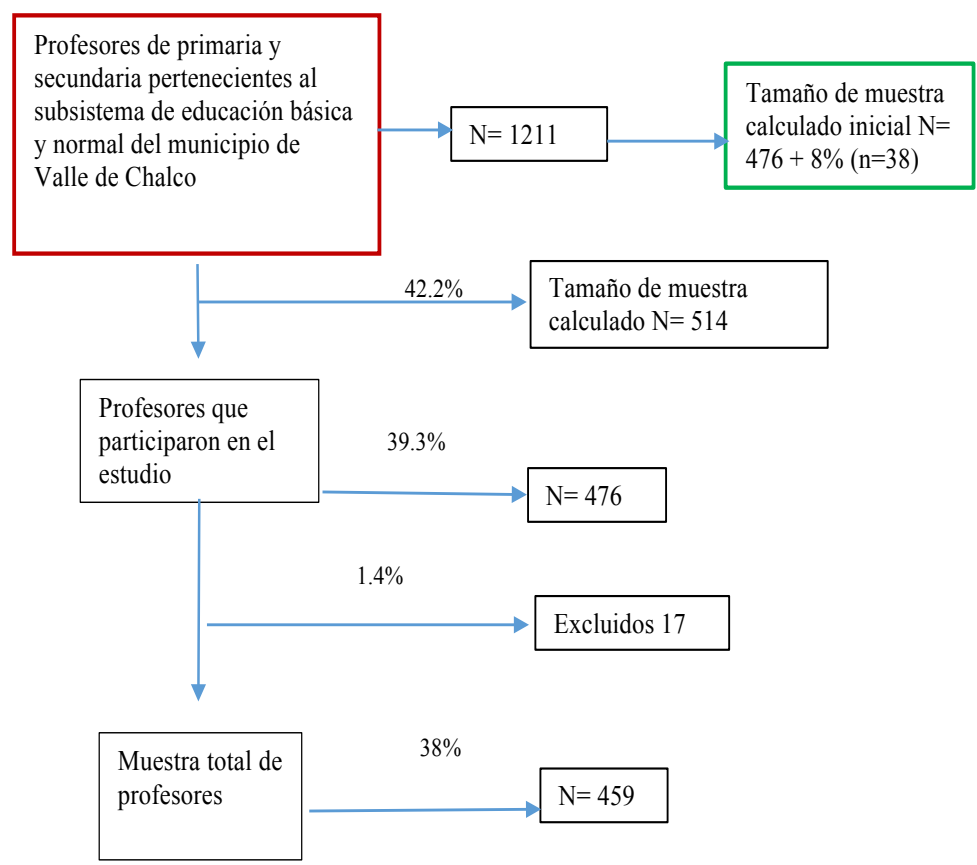

Fuente: elaboración propia a partir de los datos proporcionados por la Casa Sindical del SMSEM perteneciente a la Subdirección Regional Amecameca.

De la población de profesores sólo a 39.3 por ciento fue posible muestrear y corresponde a un total de 476 profesores. Se eliminaron 17 test de profesores que no cumplimentaron uno de los cuestionarios o por faltar información relevante, el porcentaje correspondiente es de 1.4 por ciento de la población. El muestreo final fue de 459 profesores que representan 38 por ciento de la población (1211), el número de profesores que faltaron para completar la muestra fue de 55 que representa aproximadamente 4.5 por ciento del total. 
El tamaño de la muestra para los profesores de primaria es de 296 (de 696), que representa 42.5 por ciento y de secundaria 218 (de 515) que es 42.3 por ciento dando un total de 514 profesores para la muestra. De estas proporciones 39.1 por ciento $(272 / 696)$ y 36.3 por ciento $(187 / 515)$ de los profesores de primaria y secundaria respectivamente están incluidos en este estudio representando 89.3 por ciento (459/514) del total de la muestra seleccionada. El número de profesores que faltó incluir fue de $55 \mathrm{y}$ representa 11.9 por ciento del tamaño de la muestra. El tamaño de muestra calculado inicial fue de 476 profesores

\section{INSTRUMENTOS}

Se integró para su aplicación un cuestionario elaborado exprofeso para recabar información sobre los datos sociodemográficos del profesor, el inventario de Burnout de Maslach-Ed para docentes MBI-Ed (por sus siglas en inglés Maslach Burnout Inventory-Educators). Los inventarios de depresión y ansiedad de Beck. El cuestionario de datos sociodemográficos y los test fueron aplicados de manera colectiva y autoadministrada a los profesores. El tiempo estimado para dar indicaciones, responder el cuestionario y los test en promedio fue de 50 minutos.

\section{Inventario de Burnout de Maslach (MBI-Ed)}

El inventario de Burnout de Maslach para educación (MBI-Ed) (Seisdedos, 1997) fue diseñado para valorar el SBO en sus tres aspectos fundamentales y donde cambian la palabra cliente por alumno. En la versión utilizada en esta investigación se sustituye la palabra "quemado" que corresponde al item ocho de la prueba por fatiga mediante un estudio piloto realizado previamente, los resultados no son incluidos en este estudio. El test está compuesto por 22 items y consta de tres subescalas divididas en las siguientes categorías: i) agotamiento emocional (alfa de cronbach 0.90), ii) despersonalización (alfa de cronbach 0.76) y iii) realización personal (alfa de cronbach 0.76). Para su evaluación e interpretación se integran a partir de su distribución en tercios: bajo, medio y alto (Maslach y Jackson, 1996). Para la distribución de los valores se consideraron los percentiles 33 y 66 de los puntajes totales por subescala.

Los criterios para designar un caso de Burnout de acuerdo a Maslach y Jackson (1981) son los siguientes: 
1. Grado alto de Burnout: cuando el puntaje de las dos subescalas de agotamiento emocional (AE) y el de despersonalización (D) son altas y el de realización personal (RP) es baja.

2. Grado medio de Burnout: el resultado promedio de las tres subescalas es medio.

3. Grado bajo de Burnout: el resultado de las subescalas de agotamiento emocional (AE) y despersonalización (D) son bajos y el puntaje en la subescala de realización personal (RP) es alto. Los cortes estimados para esta muestra se determinaron a partir del percentil 33.3 y 66.6 (Tabla 1).

Tabla 1: Índice del Síndrome de Burnout

\begin{tabular}{lrrr}
\hline Subescalas MBI & Bajo (1) & Medio (2) & Alto (3) \\
\hline Agotamiento emocional (AE) & $\leq 14$ & $15-23$ & $\geq 24$ \\
Despersonalización (D) & $\leq 2$ & $3-5$ & $\geq 6$ \\
Realización Personal (RP) & $\geq 35$ & $34-29$ & $\leq 28$ \\
\hline
\end{tabular}

Fuente: elaboración propia. Distribución de los datos de acuerdo al percentil 33.3 y 66.6 Alto $=$ tercio superior. Medio $=$ tercio medio. Bajo $=$ tercio inferior.

\section{El Inventario de Depresión de Beck (BID)}

Fue desarrollado en 1961 para evaluar específicamente la severidad de los síntomas de depresión. Fue traducido y estandarizado al español para la población mexicana en 1998. Es un test autoaplicable con un alfa de cronbach de 0.87 , el cual consta de 21 ítems graduando las respuestas en una escala numérica tipo Likert en donde la puntuación va de 0 a 3 . Se calificó de acuerdo a los criterios establecidos en la versión mexicana del Inventario de Depresión de Beck (BDI por sus siglas en inglés) en una escala de 0-63 puntos, cada respuesta con valor de 0-3 puntos. Se obtuvo la suma total y se interpretó de la siguiente manera: $\leq 9$, sin síntomas depresivos; de 10-16, sintomatología leve; de 17-29, moderada; $\mathrm{y} \geq 30$ puntos, sintomatología depresiva severa.

\section{El Inventario de Ansiedad de Beck (BIA)}

Fue desarrollado en 1988 para evaluar específicamente la severidad de los síntomas de ansiedad. El Inventario de Ansiedad de Beck fue traducido y estandarizado al español. Se calificó de acuerdo a una escala de 0-63 puntos, cada respuesta con valor de 0-3 puntos. Aquellos con $\leq 7$, sin sintomatología de ansiedad; de 8-15, sintomatología leve; de 16-25, moderada; $\mathrm{y} \geq$ 26, ansiedad severa (Tabla 2). 
Tabla 2: Porcentaje de profesores de acuerdo a las subescalas del Síndrome de Burnout

\begin{tabular}{lrrr}
\hline Subescalas MBI & Bajo & Medio & Alto \\
\hline Agotamiento emocional (AE) & 46.8 & 28.3 & 24.8 \\
Despersonalización (D) & 39.2 & 22.4 & 38.3 \\
Realización Personal (RP) & 62.1 & 24.2 & 13.7 \\
\hline
\end{tabular}

Fuente: elaboración propia. Distribución de los datos de acuerdo al percentil 33.3 y 66.6 Alto $=$ tercio superior. Medio $=$ tercio medio. Bajo $=$ tercio inferior.

El periodo de aplicación de los instrumentos fue del 1 de noviembre de 2014 al 1 noviembre de 2015.

\section{Procedimiento}

Previo a la aplicación de los cuestionarios, por lo menos una semana antes, se solicitó una reunión con los supervisores con la finalidad de obtener su permiso y consentimiento verbal. Para facilitar la aplicación de los test se eligió el día de reunión del consejo técnico. Dicho consejo se reúne el último viernes de cada mes. Estas reuniones tienen lugar en algún centro escolar perteneciente a la supervisión escolar y es determinado por el supervisor. En ellas se reúnen todos los docentes pertenecientes a la zona escolar en sus respectivas jornadas laborales.

Debido a la situación de tiempo, espacio, organización de las escuelas, necesidades administrativas y/o académicas de las instituciones, la aplicación de los cuestionarios se realizó bajo las siguientes condiciones:

1. En la primera de ellas los instrumentos de evaluación se aplicaron el día del consejo técnico escolar. Se acudió a la hora acordada previamente por el supervisor y ese día en coordinación con los directores se eligieron a 50 profesores del turno matutino de diferentes escuelas para participar en el estudio y 37 correspondientes al turno vespertino. El lugar para la aplicación de los test fue la biblioteca escolar donde fueron reunidos los profesores.

2. El segundo procedimiento de aplicación se realizó el día de la reunión del consejo técnico. Se acudió a la hora indicada por el supervisor y por conveniencia de las necesidades de espacio y tiempo de las escuelas se decidió que la aplicación de los cuestionarios fuera en cada una de las aulas donde estaban concentrados los profesores por escuela. Esta forma se aplicó a una zona de secundarias donde fueron evaluados 108 profesores de ambos turnos, 55 correspondientes al turno matutino y 53 correspondientes al turno vespertino. Una zona de primaria 
donde fueron evaluados 154 profesores, 90 del turno matutino y 64 del vespertino.

3. La aplicación de los test se realizó en las escuelas seleccionadas de la zona escolar correspondiente, asistiendo el aplicador a ambos turnos, en el turno matutino a las 12 horas y en el vespertino a las 17 horas en diferentes días, otorgando los directores un espacio de 60 minutos para que los profesores pudieran responder los cuestionarios sin ningún contra tiempo. En esta zona se evaluaron a 87 profesores, 50 del turno matutino y 37 del vespertino.

4. En otra zona escolar de primarias; en una escuela se aplicaron 40 cuestionarios, 20 correspondieron al turno matutino y 20 al turno vespertino, aquí, hubo una variación del procedimiento, se aplicaron los test en las escuelas a las nueve horas en el turno matutino y a las 13:30 horas en el vespertino y con el consentimiento del director se acudió a cada salón de clases para explicar a los docentes la finalidad de la investigación y que pudieran contestarlo dentro del aula con los alumnos en clase. El tiempo que les tomó a los profesores para contestarlo fue aproximadamente de dos horas y media. Una de las indicaciones que se les dio fue que el aplicador pasaría a la hora y a las dos horas para saber si se necesitaban algo, si tenían algunas dudas sobre los test o si ya había terminado. Después de dos horas de que los profesores comenzaron a contestar los test el aplicador paso a cada salón para saber si ya habían terminado de contestar los cuestionarios. Sin embargo, la mayoría de ellos no lo había terminado. Este procedimiento fue incómodo para los profesores ya que tenían que estar atendiendo a los alumnos y contestarlos, por lo que dos profesoras de esta escuela del turno matutino entregaron en blanco los test, aclarando una de ellas que no lo cumplimentó por falta de tiempo porque tuvo que atender a padres de familia y alumnos, la segunda de ellas no hizo ningún comentario sólo lo entregó sin contestarlo.

Adicionalmente se les proporcionó la siguiente información enfatizando los siguientes puntos:

- Se realizó una breve explicación sobre la finalidad de la investigación.

- Se solicitó su consentimiento verbal para participar en la investigación.

- Se hizo énfasis sobre la confidencialidad y uso de los datos obtenidos.

- Se señaló que si decidían no participar, no habría ninguna repercusión hacia su persona o trabajo. 
- Se les explicó las características del cuestionario, de los inventarios $\mathrm{y} / \mathrm{o}$ escalas de forma general.

Al terminar de contestar los cuestionarios se les agradeció el apoyo y participación a los profesores, así como a los directivos por las facilidades otorgadas para desarrollar esta investigación.

\section{Resultados}

\section{Características sociodemográficos de la muestra}

Lo integrantes de la muestra son 459 de los cuales 276 (60.1 por ciento) son mujeres y 183 (39.9 por ciento) son hombres. Las mujeres representan la mayoría (más de la mitad) de los profesores que laboran en el municipio de Valle de Chalco en esta muestra. Las características sociodemográficas indican que la mayoría de los profesores tienen más de 30 años de edad con un rango predominante de 40 a 49 años y un promedio de 40.7 (DS * +/ 9.17) años de edad con un rango mínimo de 22 años y máxima de 64 (Tabla 3).

Tabla 3: Distribución porcentual por rango de edad

\begin{tabular}{lcr}
\hline Rango de edad & No. Profesores & Porcentaje \\
\hline $20-29$ años & 63 & 13.7 \\
30-39 años & 143 & 31.2 \\
$40-49$ años & 167 & 36.4 \\
$50-59$ años & 83 & 18.1 \\
60 años o más & 3 & 0.7 \\
\hline Fuente: elaboración propia & &
\end{tabular}

Es decir, 67.6 por ciento de los profesores está en una edad productiva laboral comprendida entre los 30 a 49 años de edad.

El estado civil predominante es el de casado (Tabla 4); 51.6 por ciento de los profesores de la muestra trabajan en ambos turnos; 15 por ciento solo trabaja en el turno vespertino y 32.5 por ciento exclusivamente en el matutino. Los profesores que laboran en ambos turnos la mayoría lo hacen en dos escuelas distintas.

El 92.4 por ciento de los profesores es de base y 3.1 por ciento tienen una plaza interina. Este porcentaje corresponde a profesores que han participado en el sistema de evaluación para acceder a una plaza y que aún no cuentan con la plaza definitiva. Por otra parte, 4.4 por ciento de los docentes tiene doble plaza pero una de ellas no es definitiva, accedieron a ella cubriendo de forma temporal esta plaza por estar vacante sin acceder a ella 
por examen de oposición. En relación a los niveles educativos en los que se distribuye el trabajo de los profesores 53.8 por ciento es en primarias, 34 por ciento trabaja en el nivel de secundaria. En menor proporción son los docentes que tiene una plaza en primaria y otra en preescolar $(0.7$ por ciento), así como también, los que tienen una plaza en primaria y otra en secundaria ( 9.4 por ciento).

Tabla 4: Características sociodemográficas y laborales de los profesores

\begin{tabular}{lrr}
\hline & $\begin{array}{c}\text { Número de } \\
\text { profesores }\end{array}$ & Porcentaje \\
\hline Estado civil & 306 & 66.7 \\
Casado & 90 & 19.6 \\
Soltero & 35 & 7.6 \\
Unión libre & 28 & 6.1 \\
Otro & & \\
Grado de estudios & 53 & 11.5 \\
Normal elemental & 37 & 8.1 \\
Lic. sin título & 254 & 55.3 \\
Lic. con título & 37 & 8.1 \\
Maestría sin título & 63 & 13.7 \\
Maestría con título & 15 & 3.3 \\
Otro & & \\
Tipo de plaza & 424 & 92.4 \\
Base & 14 & 3.1 \\
Interina & 21 & 4.5 \\
Base-interina & & \\
Niveles educativos en que laboran & 247 & 53.8 \\
Primaria & 156 & 34.0 \\
Secundaria & 3 & 0.7 \\
Preescolar-Primaria & 43 & 9.4 \\
Primaria-Secundaria & 10 & 2.2 \\
Otro (Educación básica/media o superior) & 459 & 100 \\
Total &
\end{tabular}

Fuente: elaboración propia. Número y porcentaje de profesores agrupados por sexo, estado civil, grado de estudios, tipo de plaza y niveles educativos pertenecientes al Municipio de Valle de Chalco, Estado de México.

El porcentajes entre las mujeres casadas es de 38.1 por ciento y los hombre 28.5 por ciento. El segundo rango con mayor porcentaje fue el de solteros que representa a las mujeres 12.9 por ciento y para los hombre 6.8 por ciento. La distribución de los profesores por grado de estudios, indica que un poco más de la mitad tiene estudios de licenciatura con título. El porcentaje para la mujeres con licenciatura terminada es de 36.2 por ciento 
y para los hombres de 19.2 por ciento. También en las mujeres se observa un porcentaje de titulación mayor en maestría que los hombres; 9.2 por ciento comparado con 4.6 por ciento, respectivamente.

La antigüedad en la actividad docente incluye un rango de 1 a 40 años, con una media de 16.7 (DS +/- 9.2), el rango de antigüedad en el empleo en mujeres es de 1 a 38 años con una media de 16.08 (DS +/- 0.55), años de servicio mientras que el de los hombres es de 1 a 40 años con una media 17.66 (DS+/- 0.67). Los porcentajes por años de antigüedad por rangos son muy similares, el único que tiene un rango mayor es el rango de años de servicio de 16 a 20 años de servicio con 19 por ciento.

\section{SíndRome DE Burnout}

La clasificación de los profesores en las subescalas se realizó de acuerdo a los percentiles 33 y 66 (ver Tabla 1). En las subescala de agotamiento emocional se observan que la mayoría de los profesores presenta un grado de agotamiento emocional bajo, $46.8,28.3$ por ciento en el grado medio y 24.8 por ciento en un grado alto (Tabla 4 ).

Los porcentajes para la subescala de despersonalización fue ligeramente mayor en el nivel bajo con 39.2 por ciento comparando con el nivel alto que fue de 38.3 por ciento.

En la subescala de realización personal el porcentaje más alto fue en el nivel bajo 62.1 por ciento que representa inversamente en la escala un mayor nivel de realización personal de los profesores. Un nivel alto en la escala de RP de Burnout le corresponde 13.7 por ciento que equivale a un nivel bajo de realización personal.

En síntesis, la proporción de SBO en un nivel bajo para la subescala de agotamiento emocional es alta ( 47 por ciento) casi la mitad de la muestra. También, esta situación se observa en la subescala de despersonalización (39 por ciento). Si bien, en un sentido estricto están en el límite representan una población de riesgo en el desarrollo del SBO moderado y severo. En la subescala de realización personal la mayoría de los profesores obtuvo puntajes altos (62 por ciento), lo que indica que existe en esta muestra de profesores una alta satisfacción con respecto a los logros en su trabajo.

\section{COMORBILIDAD ENTRE SÍNDROME DE BURNOUT, DEPRESIÓN Y ANSIEDAD}

En el análisis de la comorbilidad entre el Burnout, la ansiedad y la depresión considerando solo un factor, es decir, excluyendo la presencia de algún otro factor para cada morbilidad, el que mayor prevalencia presenta 
es la ansiedad con 30.9 por ciento, seguido del SBO con 20.8 por ciento y en menor medida comparado con los otros dos la depresión con 10.6 por ciento (Figura 2). En esta interacción solo se muestran los casos que tienen algún grado de SBO, de ansiedad o depresión; los que no tienen alguna condición quedan excluidos de la figura, el tamaño de la muestra con alguna comorbilidad es de 324 profesores (58.7 por ciento) y 135 (41.3 por ciento) maestros sin morbilidad.

Figura 2: Comorbilidad entre síndrome de Burnout, ansiedad y depresión

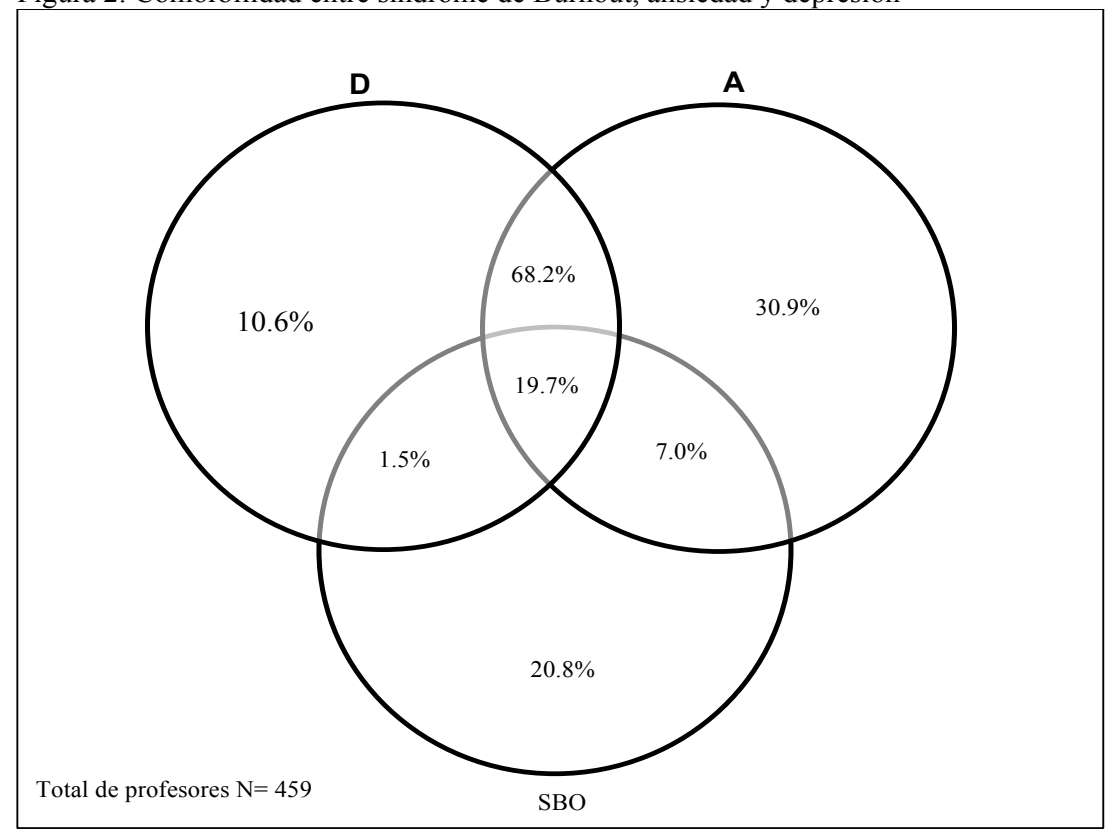

Fuente: elaboración propia. Diseño factorial $3 \times 2 \times 2$. Síndrome de Burnout (SBO), Depresión (D) y Ansiedad (A). El total de la muestra es de 459 profesores de la cual 135 ( 41.3 por ciento) no presentan alguno de los tres trastornos. El análisis de comorbilidad incluye a 324 (58.7 por ciento) maestros con alguna de las tres condiciones en algún grado de severidad (SBO, A y/o D).

La comorbilidad entre ansiedad y depresión es alta, representa 68.2 por ciento, aproximadamente siete de cada diez profesores presenta ansiedad y depresión. La relación entre ansiedad y depresión con el SBO son relativamente bajas, SBO y ansiedad siete por ciento y SBO y ansiedad 1.5 por ciento que es la más baja comparada con el de ansiedad y depresión.

La comorbilidad en los profesores de esta muestra en la que se analiza el SBO, la ansiedad y la depresión es alrededor de 18 por ciento es decir, aproximadamente dos profesores de cada diez presentan las tres condiciones que en términos generales podemos considerarla relevante. 
Cuando se analiza la prevalencia de estos trastornos de forma independiente se puede observar que éstas son mayores que cuando se analizan en interacción, es decir, se observa una prevalencia del SBO de 25.9 por ciento, de la depresión de 28.8 por ciento y de la ansiedad de 52.3 por ciento (Figura 3).

Figura 3: Prevalencia de los trastornos

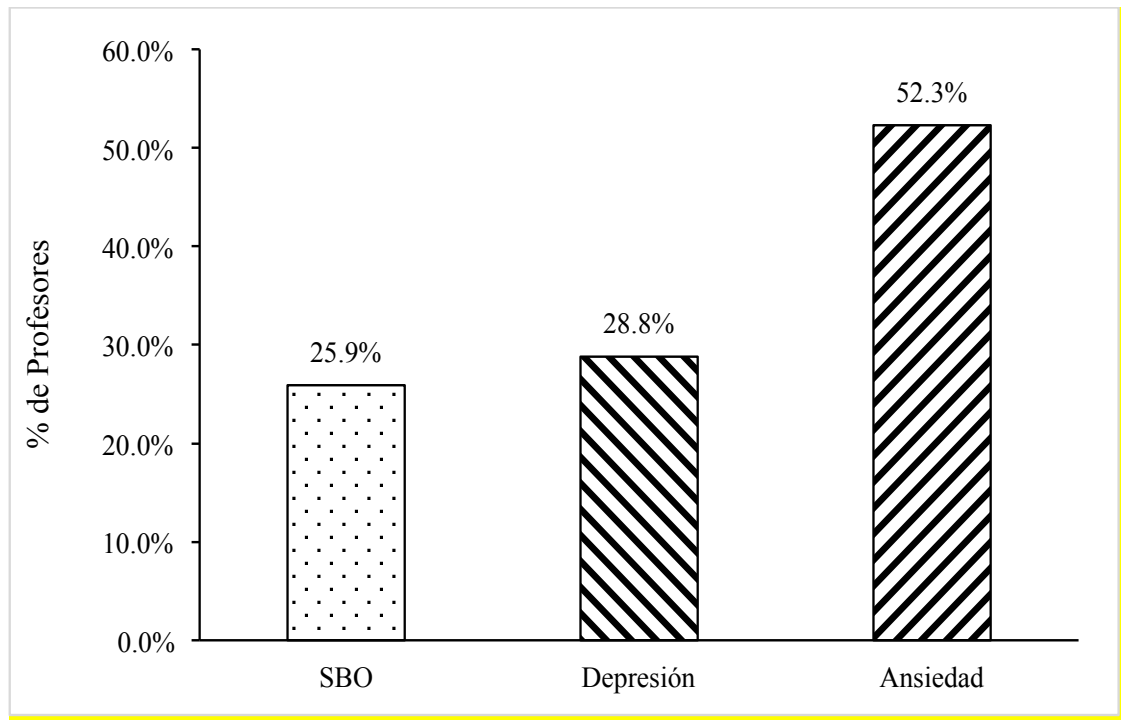

Fuente: elaboración propia. Este gráfico ilustra las prevalencias de los trastornos de forma independiente. Se observa una mayor prevalencia de ansiedad, seguido por la depresión en profesores.

Por otra parte, el grado de relación entre las variables ansiedad y depresión es alta, la varianza explicada entre este grupo es 65.8 por ciento, es positiva y estadísticamente significativa, es decir, a medida que se incrementa el puntaje en la ansiedad se incrementa el puntaje de depresión al menos en 66 por ciento de los profesores de esta muestra (Tabla 5).

La relación entre depresión y la subescala de agotamiento emocional es una correlación media, significativa $(\mathrm{p}<0.01)$ y positiva la varianza explicada es de 0.42 , a medida que se incrementa el puntaje en la escala de depresión aumenta el de la subescala $\mathrm{AE}$ al menos en 42 por ciento de los profesores.

La depresión y la despersonalización (DES) muestran una relación baja, positiva y significativa $(\mathrm{p}<0.01)$. La varianza explicada es alrededor de 0.17 . Esto sugiere que a medida que se incrementa el puntaje de depresión se incrementa el de despersonalización al menos en 17 por ciento de los maestros. 
Tabla 5: Correlación entre índice de ansiedad, depresión y subescalas del síndrome de Burnout

\begin{tabular}{|c|c|c|c|c|c|}
\hline \multicolumn{2}{|c|}{ Correlac } & IA & $\mathrm{AE}$ & DES & RP \\
\hline \multicolumn{6}{|c|}{ Rho de Spearman } \\
\hline \multirow[t]{3}{*}{ ID } & Coeficiente de & $0.658^{*}$ & $0.417^{*}$ & $0.165^{* *}$ & $-0.274^{*}$ \\
\hline & Sig. & 0.000 & 0.000 & 0.000 & 0.000 \\
\hline & $\mathrm{N}$ & 459 & 459 & 459 & 459 \\
\hline \multirow[t]{3}{*}{ IA } & Coeficiente de correlación & & $0.440^{*}$ & $0.176^{*}$ & $-0.288^{*}$ \\
\hline & Sig. (bilate & & 0.000 & 0.000 & 0.000 \\
\hline & $\mathrm{N}$ & & 459 & 459 & 459 \\
\hline
\end{tabular}

* La correlación es significativa al nivel 0.01 (bilateral).

Fuente: elaboración propia. Índice de depresión (ID), índice de ansiedad (IA). Subescalas

del síndrome de Burnout: agotamiento emocional (AE), despersonalización (DES) y

realización personal $(\mathrm{RP})$.

En relación a la depresión y realización personal (RP) se observa una correlación baja, negativa y significativa $(\mathrm{p}<0.01)$, la varianza explicada es alrededor de 0.27. Es decir, a medida que se incrementa el índice de depresión entre los profesores disminuye el puntaje en la subescala RP al menos en 27 por ciento de los maestros.

La relación entre índice de ansiedad (IA) y la subescala de AE es media positiva y significativa ( $\mathrm{p}<0.01$ ), la varianza explicada es de 0.44 . A medida que se incrementa el IA aumenta el de la subescala de agotamiento emocional en 44 por ciento de los profesores.

Para el caso de ansiedad y DES se observa una relación baja, significativa y positiva, la varianza total explicada es alrededor de 0.18 , lo que sugiere que a medida que incrementa el IA se incrementa la DES en 18 por ciento de los maestros.

Finalmente, la correlación entre IA y la realización personal es significativa, baja y negativa. La varianza explicada es alrededor de -0.29 lo que indica, que a medida que se incrementa el IA el de RP disminuye.

\section{Comorbilidad en el Síndrome de Burnout de aCuerdo al SeXo}

De los 459 profesores de la muestra 29.4 por ciento (135) no presentan SBO, depresión o ansiedad. En el otro grupo de profesores 70.6 por ciento (324) presentan alguna comorbilidad, es decir, SBO, depresión y/o ansiedad. El número de mujeres en la muestra es de 201 (43.8 por ciento) y el de los hombres es de 123 (26.8 por ciento) con alguna comorbilidad (Figura 4). 
Comorbilidad entre sindrome de Burnout, depresión y ansiedad en una muestra .../F. SÁNCHEZ y J.J. VELASCO

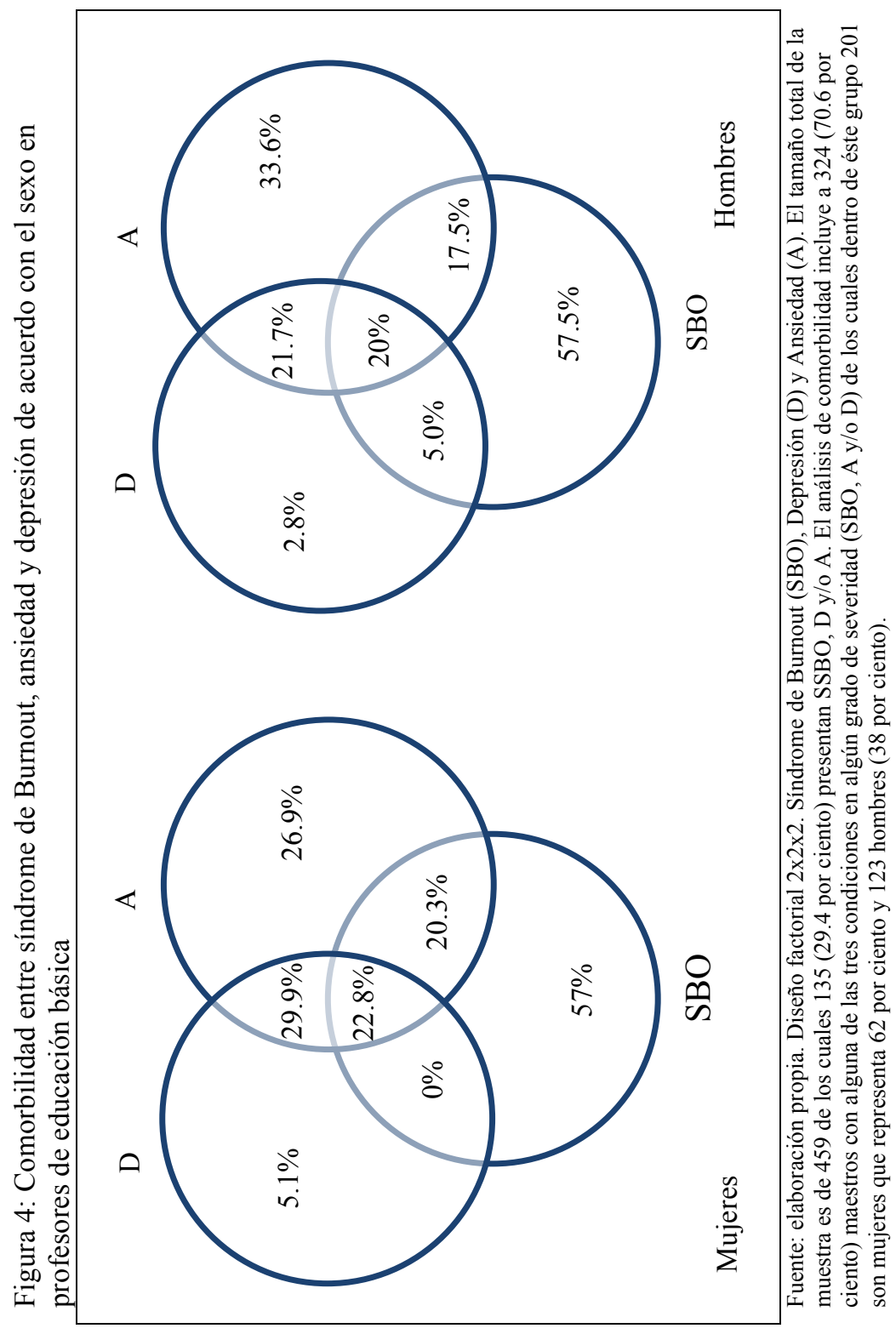


Los resultados de la comorbilidad entre SBO, depresión y ansiedad de acuerdo al sexo es el siguiente: para el grupo de mujeres el SBO presenta una prevalencia de ansiedad de 26.9 por ciento, seguido por el de depresión 5.1 por ciento en mujeres. La relación entre depresión y ansiedad es de 29.9 por ciento.

En las proporciones correspondientes para los hombres se observa un incremento en la ansiedad (33.6 por ciento) y una reducción en los porcentajes de depresión de 2.8 por ciento comparados con el de las mujeres. La comorbilidad entre ansiedad y depresión en el sexo masculino (21.7 por ciento) es menor que el de las mujeres.

Los porcentajes en los maestros con SBO de acuerdo al sexo es el siguiente: la proporción de mujeres con SBO es de 57 por ciento, Ansiedad y SBO es de 20.3 por ciento. No existe comorbilidad entre depresión y SBO.

Para el sexo masculino 57.5 por ciento presenta SBO, la comorbilidad entre SBO y ansiedad es de 17.5 por ciento un poco menos que el de las mujeres, mientras que para la relación con la depresión se incrementa a cinco por ciento comparado con el sexo femenino.

El porcentaje de mujeres con SBO, A y D es de 22.8 por ciento, ligeramente mayor que el de los hombres con una comorbilidad de 20 por ciento.

La diferencia entre las proporciones del SBO de acuerdo al nivel educativo y a la presencia de depresión y/o ansiedad es estadísticamente significativa (estadístico exacto de Fisher $\mathrm{p}<0.01$ ) con un coeficiente de contingencia mediano de 0.45 con una significancia de $\mathrm{p}<0.01$.

\section{Comorbilidad en el SíndRome de Burnout de ACUERdo AL NIVEL EDUCATIVO}

De los 459 profesores de la muestra 29.4 por ciento (135) no presentan síndrome de Burnout, depresión o ansiedad. En el otro grupo de profesores 70.6 por ciento (324) presentan alguna comorbilidad, es decir, SBO, depresión y/o ansiedad. El número de profesores de primaria en la muestra es de 197 (42.9 por ciento) y el de secundaria es de 127 (27.6 por ciento) con alguna comorbilidad.

Los resultados de la comorbilidad entre SBO, depresión y ansiedad de acuerdo al nivel educativo en que tienen su plaza principal es el siguiente: para el grupo de primaria SSBO se presenta una prevalencia de ansiedad de 31.2 por ciento, seguido por el de depresión seis por ciento en los profesores de primaria. La relación entre depresión y ansiedad en éste nivel es de 29.3 por ciento (Figura 5). 
Comorbilidad entre sindrome de Burnout, depresión y ansiedad en una muestra .../F. SÁNCHEZ y J.J. VELASCO

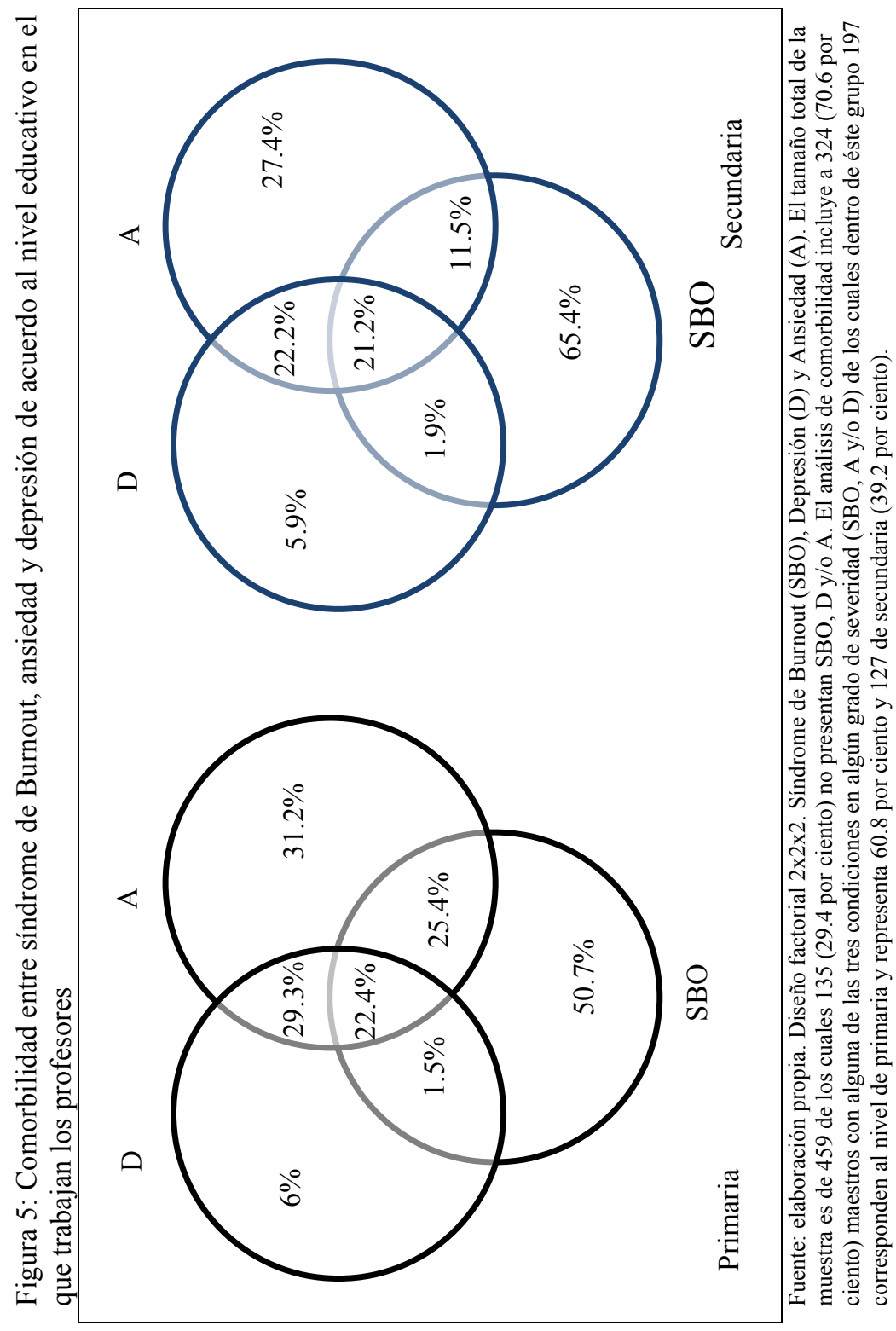


Las proporciones correspondientes para los profesores de secundaria SSBO se observa un disminución de la ansiedad (27.4 por ciento) y un porcentaje equivalente en depresión (5.9 por ciento) con los de primaria. La comorbilidad entre ansiedad y depresión en el grupo de secundaria es menor que el de los de primaria.

Los porcentajes en los maestros con SBO del nivel de primaria es el siguiente: la proporción de profesores con SBO es de 50.7 por ciento, Ansiedad y SBO es de 25.4 por ciento mientras que para depresión con SBO es 1.5 por ciento.

Para el nivel de secundaria 65.4 por ciento presenta SBO el cual es mayor que el de primarias, la comorbilidad entre SBO y ansiedad es de 11.5 por ciento menor que el de los profesores de primaria, mientras que para la relación con la depresión se mantiene casi sin cambios (5.9 por ciento) comparado con el de primaria.

El porcentaje de profesores de primaria con SBO, A y D es de 22.4 por ciento, ligeramente mayor que el de los secundaria con una comorbilidad de 21.2 por ciento.

La diferencia entre las proporciones del SBO de acuerdo al nivel educativo y a la presencia de depresión y/o ansiedad es estadísticamente significativa (estadístico exacto de Fisher $\mathrm{p}<0.01$ ) con un coeficiente de contingencia mediano de 0.5 con una significancia de $\mathrm{p}<0.01$.

\section{Conclusiones}

Dentro del contexto educativo y escolar a la salud del profesor no se le da mucha importancia. Sólo con revisar brevemente la Ley Federal del Trabajo y la Ley General de Salud nos podemos dar cuenta fácilmente. Sin embargo, existen enfermedades o trastornos psicosociales que afectan su desarrollo psicológico, emocional y personal del maestro. Estos repercuten en su desempeño, calidad de vida, relaciones familiares y sociales. Los efectos de estos trastornos (el SBO, la depresión y la ansiedad) son tan sutiles y en ocasiones a largo plazo que desde el punto de vista social, cultural, económico y político se le da muy poca importancia, pero eso no significa que no estén presentes y que no repercutan en su salud y bienestar.

Los resultados obtenidos en este estudio muestran una prevalencia alta para el síndrome de Burnout y la ansiedad, y en menor proporción la depresión aunque es mayor que la reportada en la población general. Por otra parte, al considerar los tres trastornos en forma independiente más de la mitad de los profesores presentan entre SBO, ansiedad o depresión. En este sentido, encontramos proporciones mayores en el análisis individual de los 
trastornos como el Burnout, la depresión y la ansiedad y estos porcentajes disminuyen cuando se analiza su interacción o comorbilidad.

El análisis de las comorbilidades también ayuda a dimensionar estos trastornos como un problema de salud en los profesores. La comorbilidad entre el SBO y la depresión es baja y esta se incrementa ligeramente cuando se analiza la relación entre SBO y ansiedad. Observamos una comorbilidad alta entre la depresión y la ansiedad. Esto indica una tendencia mayor entre estos dos trastornos, es decir la mayoría de los profesores presentan ansiedad y depresión en forma simultánea, lo cual sugiere un trastorno mixto. Aunque en este estudio no se analiza la severidad de los trastornos, se incluye a todos aquellos que presentan un problema de acuerdo a la clasificación propuesta por cada uno de los instrumentos. Finalmente, la presencia del síndrome de Burnout, depresión y ansiedad simultáneamente en los profesores es alta en una proporción de 20 por cada 100 profesores. A nuestro entender y con respecto a la literatura revisada, este es el primer trabajo, que describe la coincidencia de estos trastornos en un mismo individuo o grupo.

Las diferencias por sexo entre el SBO, depresión y ansiedad presentan algunos cambios. La prevalencia de ansiedad en maestros es mayor que en las maestras. En los otros parámetros se observa una mayor prevalencia en las mujeres en comorbilidad entre depresión y ansiedad, entre SBO y ansiedad, y entre depresión, ansiedad y SBO. Estas prevalencias sugieren en los profesores del municipio de Valle de Chalco Solidaridad una mayor vulnerabilidad de las mujeres a presentar estos trastornos que los hombres. Los maestros por otra parte, parecen ser más vulnerables a los trastornos de ansiedad.

Las prevalecias por nivel educativo en este municipio son: i) el SBO es mayor en el nivel de secundaria, la ansiedad es mayor en los profesores de primaria y la depresión es casi la misma en ambos niveles. ii) La comorbilidad entre SBO y ansiedad es mayor en los profesores de primaria. iii) La prevalencia entre SBO y depresión es casi igual en ambos niveles y la comorbilidad entre ansiedad y depresión es mayor en los profesores de primaria. iv) La prevalencia entre los tres trastornos es casi igual en ambos niveles. Al parecer las condiciones a las que está expuesto el profesor de primaria lo hacen más vulnerable a estos trastornos.

Por otra parte, la correlación establecida entre las subescalas del SBO y los índices de depresión, y ansiedad indican correlaciones positivas, es decir, a medida que aumenta el puntaje en la escala de agotamiento se incrementa el puntaje de depresión y de ansiedad, lo mismo ocurre con la 
subescala de despersonalización. Finalmente se observa una correlación negativa con la subescala de realización personal, a mayor puntaje en la escala de ansiedad o depresión menor realización personal. Es importante, resaltar que existe una relación importante entre estas subescalas, la ansiedad y la depresión.

En este sentido la evidencia apunta a que los trastornos mencionados están relacionados y que pueden reflejar un mismo problema, principalmente entre la ansiedad, depresión y la subescala de agotamiento emocional del Burnout.

Sin embargo, también consideramos que estos trastornos pueden ser diferenciados clínicamente, el estudio de la comorbilidad entre estos padecimientos nos permite identificar aquellos casos que presentan uno, ambos o los tres trastornos en los docentes de forma simultánea, facilitando con esto la diferenciación de los trastornos o cuando se presenta más de uno.

Este punto es importante, en primer lugar, porque al parecer el estudio de las prevalencias de forma independiente se sobreestiman reportándose proporciones mayores. En segundo lugar, cuando se analizan en forma integrada, es decir, excluyendo los casos que solo presentan SBO, depresión o ansiedad de aquellos que presentan ambos o los tres trastornos de forma simultánea, las proporciones se redistribuyen o disminuyen de manera importante. De ahí el hecho de que sea relevante desde un punto de vista epidemiológico y clínico estimar en forma simultánea los tres padecimientos para realizar un diagnóstico diferencial y no sobrevalorar las proporciones tasadas de los trastornos en cuestión.

A partir de los datos presentados, se puede inferir que es fácil confundir las enfermedades descritas o que se sobrepongan estos trastornos, Situación que hace difícil realizar un diagnóstico adecuado y oportuno por parte de los especialistas y es más complicado que los profesores atiendan estos problemas porque es difícil que reconozcan los síntomas, minimizando en ocasiones las enfermedades.

También, el reconocimiento del Burnout enfrenta varias dificultades, una de ellas es que no es reconocido como un trastorno o enfermedad en México desde un punto de vista legal y social, aunque la OMS y la OIT lo consideran como un problema de salud laboral importante. Tampoco se incluye como diagnóstico en la clasificación internacional de enfermedades (CIE-10) o en la clasificación de los trastornos mentales (DSM-V). En parte, por la gran similitud que tiene con los cuadros o tipos de depresión, incluso es considerada como parte de un subtipo de depresión por algunos investigadores. Por otra parte, existe una estimación negativa con respecto 
al conocimiento y reconocimiento de los trastornos depresivos y ansiosos por parte de las personas que los padecen, así como de la sociedad, lo que dificulta su estudio y la identificación de la magnitud de su prevalencia.

Sobre la base de los resultados de la investigación, es posible señalar algunas de las área prioritaria para el sistema de salud y la Ley Federal del Trabajo en las que es necesario se revalore el cuadro de enfermedades de riesgo profesional para los maestros y se creen condiciones para el desarrollo de un sistema de diagnóstico adecuado, así como la instrumentación de medidas encaminadas a la prevención de estos trastornos en el ámbito laboral.

Una de las limitaciones de este estudio es que no se incluyeron para su análisis aquellos factores o circunstancia que favorecen la aparición de estos trastornos en el ámbito laboral. Es importante identificar específicamente ciertos riesgos relacionados a estos trastornos y que comprometen la salud del profesor en el contexto laboral y proponer medidas preventivas acordes a la problemática.

Una segunda limitación es que no se estudia y discute la comparabilidad del Inventario de Maslach con otros estudios en donde se utilizan otros instrumentos para evaluar el Burnout ya que escapa a los fines de este estudio, pero que sin duda pueden aportar elementos de discusión y análisis sobre la disyuntiva si son trastornos diferentes o son uno solo.

\section{REFERENCIAS BIBLIOGRÁFICAS}

Aronsson, G., Theorell, T., Grape, T., Hammarström, A., Hogstedt, C., Marteinsdottir, I., Skoog, I., Träskman-Bendz, L., Hall, C., 2017, "A systematic review including meta-analysis of work environment and Burnout symptoms" in $B M C$ Public Health. 17(1):264

Barraza-Macias, A., 2011, Satisfacción laboral y sindrome de Burnout en profesores de educación primaria. Estrés, Burnout y bienestar subjetivo. Disponible en http://redie.mx/librosyrevistas/libros/estres_burnout_y_bienestar_sujetivo.pdf (consultado el 16/10/2015).

Bianchi, R., Schonfeld, IS., Laurent, E., 2015a, "Is it Time to Consider the "Burnout Syndrome” A Distinct Illness?", in Front Public Health. 8 (3), pp. 158-160.

Bianchi, R., Schonfeld, IS., Laurent, E., 2015b, "Burnout-depression overlap: a review", in Clin Psychol Rev, vol. 36, pp. 28-41.

Bianchi, R., Verkuilen, J., Brisson, R., Schonfeld, IS., Laurent, E., 2016, "Burnout and depression: Label-related stigma, help-seeking, and syndrome overlap", in Psychiatry Res. 30 (245), pp. 91-98 
Borrelli, I. et al., 2014, "Working conditions and mental health in techers a preliminary study", in Occupational Medicine, vol. 64, pp. 530-532.

Caballero-Domínguez, C., González-Gutiérrez, O., y Palacio Sañudo, 2015, "Relación del burnout y el engagement con depresión, ansiedad y rendimiento académico en estudiantes universitarios", en Salud Uninorte, pp. 59-69.

Chambers, R. y Belcher, J., 1993, "Comparision of the health and life of general practitioners and teachers", in British Journal of General Practice, vol. 43, pp. 378-382.

Chong, E. Y., 2010, "Subjetive health compaints of teachers from primary and secondary schools in Hong Kong", in International Journal of Occupational Safety and Ergonomics, 16(1), pp. 23-39.

Claro, S. y Bedegral , P., 2003, "Mental health satatus of teachers in 12 schools of Puente Alto, Santiago, Chile", in REv Med Chil, 131(2), pp. 159-167.

Feuerhahn, N. et al., 2008, "Emotional exhaustion and cognitive performance in apparently Healthy teachers: A longitudinal multi-source study", in Stress Health, 29(4), pp. 297-306.

Fischer, R. y Boer, D., 2011, "What is more important for national well-being: money or autonomy? A meta-analysis of well-being, burnout, and anxiety across 63 societies", in J Pers Soc Psychol, 101(1), pp. 164-184.

Freudenberguer, H., 1974, "Staff burnout", in Journal of Social Issues, Issue 30, pp. 159-165.

Galvan, S., Aldrete-Rodríguez, G., Preciado, L. \& Medina Aguilar, S., 2010. Factores psicosociales y síndrome de burnout en docentes de nivel preescolar de una zona escolar de Guadalajara. Revista de Educación y Desarrollo, Volumen 14, pp. $5-11$.

Juárez-García, A., Idrovo, J., Camacho-Ávila, A. y Placencia-Reyes, O., 2014, "Síndrome de Burnout en población mexicana: una revisión sitemática", en Salud Mental, vol. 37, pp. 159-176.

Kidger, J. et al., 2016, "Teachers's wellbeing and depresive syntoms, and associated risk factors: A large cross sectional study schools", in Journal of Affective Disorders, vol. 192, pp. 76-82.

Kohn, R. et al., 2005, "Los trastornos mentales en América Latina y el Caribe: asunto prioritario para la salud pública", in Rev Panam Salud Públial Pan Am J Public Health, 18(4/5), pp. 229-240.

Kovess-Masfésty, V. et al., 2006, "Do teachers have more health problems? Result from a French cross-sectional survey", in BMC Public Health, vol. 6, p. 101.

Lindwall, M., Gerber, M., Jonsdottir, IH., Börjesson, M., Ahlborg, G. Jr., 2014, "The relationships of change in physical activity with change in depression, anxiety, and burnout: a longitudinal study of Swedish healthcare workers", in Health Psychol. 33(11), pp. 1309-1318. 
Lopes Cardozo, B., Gotway Crawford, C., Eriksson, C., Zhu, J., Sabin, M., Ager, A., Foy, D., Snider. L., Scholte, W., Kaiser, R., Olff, M., Rijnen, B., Simon, W., 2012, "Psychological distress, depression, anxiety, and burnout among international humanitarian aid workers: a longitudinal study", in PLoS One. 7(9) p. e44948.

Marin, MF., Lord, C., Andrews, J., Juster, RP., Sindi, S., Arsenault-Lapierre, G., Fiocco, AJ., Lupien, SJ., 2011, "Chronic stress, cognitive funtion and mental health", in Neurobiol Learn Mem, 96(4), pp. 583-595.

Maslach, C. y Jackson, S. E., 1981, "The measurement of experienced burnout", in The measurement of experienced burnout, vol. 2, pp. 99-113.

Maslach, C., Leiter M. y Jackson S., 1996, Maslach Burnout Inventory Manual $(M B I)$. USA: Mind Garden

Maslach, C. y Schaufeli, W., 1993, Historical and conceptual development of burnout, en Schaufeli, W., Maslach, C. y Marek, T. (eds.) Professional Burnout: Recent developments in theory and research. USA: Taylor y Francis, pp. 99-113.

OIT, 2000, "S.O.S estrés en el trabajo: aumentan los costes del estrés en el trabajo y la incidencia de la depresión es cada vez mayor", en Trabajo, Issue 37, pp. 4-5.

Oliveira, V., y Pereira,T., 2012, “Ansiedade, depressão e burnout em enfermeiros: Impacto do trabalho por turnos", en Revista de Enfermagem Referência III Sé$\operatorname{rie}(7), 43-54$.

Pando Moreno, M. et al., 2006, "Factores psicosociales y burnout en docentes del centro universitario de ciencias de la salud", en Investigación en Salud, 8(3), pp. 173-177.

Perales, A; Chue, H., Padilla, A., Barahona, L., 2011, "Estrés, ansiedad y depresión en magistrados de Lima, Perú", en Revista peruana de medicina experimental y salud pública, vol. 28, pp. 581-588.

Pereira-Lima, K., Loureiro, SR., 2015, "Burnout, anxiety, depression, and social skills in medical residents", in Psychol Health Med. 20(3), pp. 353-62.

Schaufeli, W., 1999. "Burnout", in Firth-Cozen, J. y Payne, R. (eds.) Stress in health professionals: Psychological and organizational causes and interventions. England: Wiley, pp. 17-32.

Schaufeli, W. B. y Enzmann, D., 1998, The Burnout companion to study and practice. London: Taylor y Francis.

Scheuch, K., Eva, H. y Seibt, R., 2015, “Techers's health”, in Dtsch Arztebl Int, vol. 112, pp. 347-356.

Seisdedos, N., 1997, MBI: Inventario "Burnout” de Maslach. Síndrome del quemado por estrés laboral. Manual. Madrid: TEA.

Talarowska, M., Zajączkowska, M. y Gałecki, P., 2015, "Cognitive funtions in first-episode depression and reurrent depresive disorders", in Psychiatria Danubina, 27(1), pp. 38-43. 


\section{RESUMEN CURRICULAR DE LOS AUTORES}

Francisco Sánchez-Narváez

Licenciado en Psicología por parte de la Universidad Nacional Autónoma de México (UNAM). Maestría en Ciencias de la Educación por la Universidad del Valle de México (UVM). Doctor en Ciencias de la Educación por el Instituto Superior de Ciencias de La Educación del Estado de México (ISCEEM). Especialista en trastornos del dormir en el Instituto Mexicano de Medicina Integral de Sueño. Profesor en la Universidad Estatal del Valle de Ecatepec (UNEVE)

Dirección electrónica: fsannar01@gmail.com

Juan Jesús Velasco Orozco

Licenciado en Antropología y Maestro en antropología con énfasis en antropología social por la Universidad Autónoma del Estado de México. Doctor en antropología social por la Universidad Iberoamericana. Profesor de tiempo completo de la Facultad de Antropología de la UAEM y docente del Instituto Superior de Ciencias de la Educación del Estado de México ISCEEM.

Dirección electrónica: jujevo@gmail.com

Artículo recibido el 7 de marzo de 2016 y aprobado el 30 de agosto de 2017. 